\title{
Method
}

\section{Multiple-Locus Variable-Number Tandem Repeat Analysis Scheme for Non-O157 Shiga Toxin-Producing Escherichia coli: Focus on Serogroups O103, O121, O145, O165, and O91}

\author{
Hidemasa Izumiya*, Kenichi Lee, Nozomi Ishijima, Sunao Iyoda, and Makoto Ohnishi
}

Department of Bacteriology 1, National Institute of Infectious Diseases, Tokyo, Japan

\begin{abstract}
SUMMARY: Non-O157 Shiga toxin-producing Escherichia coli (STEC) infections are a growing concern for public health. The number of sporadic cases and outbreaks of non-O157 STEC infections have increased in recent years. Molecular subtyping is an essential tool that allows high-resolution and rapid differentiation of isolates, identification of case clusters, and detection of outbreak clusters. Multiple-locus variable-number tandem repeat analysis (MLVA) is one of the most useful typing methods for differentiating isolates that cause foodborne diseases. In Japan, serogroups O26, O111, O103, O121, O145, O165, and O91 have been frequently isolated or associated with severe cases of non-O157 STEC infections. In this study, we designed an MLVA scheme (MLVA43) for serogroups O103, O121, O145, O165, and O91 by adding 26 new loci to an MLVA scheme (MLVA17) previously developed by our group for serogroups O157, O26, and O111 using 17 loci. We found that the discriminatory power of MLVA43 was comparable to that of pulsed-field gel electrophoresis (PFGE) for serogroups O103, O145, O165, and O91, and superior to that of PFGE for O121. MLVA43 identified more profiles than did MLVA17, except for serogroup O111 with 707 isolates. The MLVA43 scheme will enable rapid detection of outbreak clusters, which will aid in implementing rapid control measures against non-O157 STEC infections.
\end{abstract}

\section{INTRODUCTION}

Shiga toxin-producing Escherichia coli (STEC) is an important etiological agent of food-borne diseases. The main symptoms of STEC infections are abdominal pain, watery diarrhea, hemorrhagic colitis, and hemolytic uremic syndrome (HUS), with potentially fatal outcomes (1). More than 3,000 cases of STEC infections are annually reported in Japan (2). Though O157 is the most commonly isolated STEC serogroup, non-O157 STEC serogroups have been increasingly isolated from sporadic cases and outbreaks in recent years. Serogroups O26, O111, O103, O121, O45, and O145 have been isolated most frequently in the United States (3), whereas serogroups O26, O111, O103, O121, O145, O165, and O91 have been most frequently isolated or associated with severe cases in Japan (2).

Molecular subtyping of STEC is essential in cluster identification and outbreak investigation. The traditional "gold standard" method for molecular subtyping is

Received March 24, 2020. Accepted May 25, 2020.

J-STAGE Advance Publication June 30, 2020.

DOI: 10.7883 /yoken.JJID.2020.095

*Corresponding author: Mailing address: Department of Bacteriology 1, National Institute of Infectious Diseases, 1-23-1 Toyama, Shinjuku-ku, Tokyo 162-8640, Japan. Tel: +81-3-5285-1111, Fax: +81-3-5285-1163, E-mail: izumiya@nih.go.jp pulsed-field gel electrophoresis (PFGE) (4). However, PFGE is time-consuming and requires high level of technical skills; therefore, multiple-locus variablenumber tandem repeat analysis (MLVA) using 17 loci has been introduced in Japan for molecular subtyping and surveillance of STEC serogroups O157, O26, and O111 $(5,6)$. MLVA differentiates the isolates based on the difference in the number of tandem repeats of several variable-number tandem repeat (VNTR) loci located on the bacterial genome (7). The target loci are amplified by multiplex PCR, and the PCR products are separated and sized using capillary electrophoresis. An allele type is assigned to each VNTR locus, based on the copy number of repeats at each locus, which is an integer value. The string of allele types for several VNTR loci constitutes an MLVA type. MLVA is advantageous because the procedure is simple and rapid, and the data obtained are portable and discrete to enable comparison among multiple laboratories (8). MLVA has been applied for typing of bacteria that cause food- and water-borne diseases, including Salmonella enterica serotypes Typhimurium and Enteritidis, Shigella sonnei, and Vibrio cholerae (9-13).

The disadvantage of MLVA is that its discriminatory power can vary among different serogroups of the target organism. In our previous study, we developed an MLVA scheme using 17 loci for STEC serogroups O157, O26, and O111 (5). Recently, another MLVA scheme was reported for serogroups O26, O111, O103, 
O121, O45, and O145 (14). However, these schemes do not include all the serogroups of O103, O121, O145, O165, and O91 that pose serious threats to public health and/or are frequently isolated in Japan. Thus, the aim of this study was to design an MLVA scheme for non-O157 STEC serogroups isolated in Japan, particularly serogroups O103, O121, O145, O165, and O91.

\section{MATERIALS AND METHODS}

Bacterial strains: A total of 707 STEC clinical isolates were used in this study. They were isolated in Japan between 2007 and 2017, but most of them were isolated in $2017(n=523)$. A total of 184 isolates (isolated between 2007 and 2016) were used for optimization of test conditions of MLVA and for comparison of the discriminatory powers of MLVA and PFGE. All the isolates were associated with either outbreaks or sporadic cases, and one representative isolate was chosen from each outbreak. An additional set of 52 isolates (36 isolates from outbreak A, and 18 isolates from outbreak B; serogroup O103) were used in cluster analyses of outbreak-related isolates. They were analyzed together with two of the 707 isolates. Patient information was anonymized in this study.

MLVA: MLVA was performed as previously described by Izumiya et al. (5) with few modifications. Primer sets for the 17 loci from the previous study are shown in Table 1 (Mix R1 and R2). The genome sequences of the strains listed in Table 2 were searched for tandem repeats in silico and 26 additional loci were identified. Primers were designed using DNASIS Pro software (Hitachi Solutions, Ltd., Japan), and their sequence specificities were examined by performing a BLAST search against the NCBI nucleotide database. The primer pairs were designed to comply with the conditions of multiplex PCR. Primer sets for the 26 additional loci are shown in Table 1 (Mix q1 and q2). One primer from each pair was fluorescently labeled for ABI PRISM GeneScan Analysis (Thermo Fisher Scientific K.K., Tokyo Japan; https://www.thermofisher. com/jp/ja/home/life-science/oligonucleotidesprimers-probes-genes/applied-biosystems-customprimers-probes.html). Template DNA was prepared using DNeasy Blood \& Tissue Kit (Qiagen K.K.Japan, Tokyo, Japan), and quantified using NanoDrop spectrophotometer (Thermo Fisher Scientific K.K.) or by heat extraction of bacterial cells. The reaction mixture for PCR contained $1 \mu \mathrm{L}$ of template DNA (30$50 \mathrm{ng}$ ) in a total volume of $15-25 \mu \mathrm{L}$. The concentration of DNA obtained by heat extraction was adjusted so that the resulting signals of the amplified products were comparable to those of DNA extracted using the kit. PCR was performed using QIAGEN Multiplex PCR Kit (Qiagen K.K.-Japan) and Platinum Multiplex PCR Master Mix (Thermo Fisher Scientific K.K.) following the manufacturer's instructions. The PCR conditions were as follows: initial denaturation at $95^{\circ} \mathrm{C}$ for 15 or 2 min (depending on the kit used); 35 cycles of denaturation at $95^{\circ} \mathrm{C}$ for $20 \mathrm{~s}$, annealing at $60^{\circ} \mathrm{C}$ for 90 $\mathrm{s}$, and extension at $72^{\circ} \mathrm{C}$ for $60 \mathrm{~s}$; and final extension at $72^{\circ} \mathrm{C}$ for $10 \mathrm{~min}$. The amplified PCR products were diluted and mixed with GeneScan 600LIZ size standard in Hi-Di Formamide (Thermo Fisher Scientific K.K.).
The diluted PCR products were sized using Applied Biosystems 3500xL Genetic Analyzer (Thermo Fisher Scientific K.K.).

PFGE: PFGE was performed for the 184 isolates according to the standardized PulseNet protocol using XbaI restriction enzyme (Roche Diagnostics GmbH, Mannheim, Germany) (15). PFGE patterns were analyzed using BioNumerics software (Applied Maths, Sint-Martens-Latem, Belgium). A dendrogram was generated based on the Dice coefficient and the unweighted pair group method with arithmetic means (UPGMA), with $1.2 \%$ positional tolerance. Isolates included in a single leaf of the dendrogram with over 95\% similarity were regarded as a PFGE partition, and are referred to as "PFGE95" hereafter.

Analysis of MLVA data: Fragment data were evaluated and converted to repeat copy numbers using GeneMapper (Thermo Fisher Scientific K.K.) and GeneMarker (SoftGenetics LLC, PA, USA) software. Data were analyzed using BioNumerics software as previously described (8). Fragments with no PCR product were assigned a repeat copy number of -2 . Simpson's diversity index (D) and 95\% confidence intervals $(\mathrm{CI})$ were calculated according to the formula described previously (16). "Moderate" and "high" indicate D values greater than 0.15 and 0.5 , respectively.

Whole genome sequencing: Complete genome sequences were obtained using MiSeq platform (Illumina, CA, USA). Genomic DNA libraries were prepared using Nextera XT DNA Sample Prep Kit (Illumina). The pooled libraries were subjected to multiplexed paired-end sequencing $(300-$ mer $\times 2)$, Minimum coverage for the analyses was $40 \times$. The short reads were assembled using SPAdes v. 3.11.1 with the "-careful" option (17).

Data availability: The assembled contigs used in this study were deposited in the DNA Data Bank of Japan. Accession numbers: BLJC01000000 for JNE080262, and BLJD01000000 for JNE160956.

\section{RESULTS}

Locus selection: We searched the complete and draft genome sequences of $E$. coli $\mathrm{O} 26(n=2), \mathrm{O} 111(n=2)$, O103 $(n=4), \mathrm{O} 121(n=1), \mathrm{O} 145(n=1), \mathrm{O} 165(n=$ $1)$, and O91 $(n=2)$ strains for tandem repeats (Table 2). Around 20 candidate loci were identified in each strain. The candidate loci were further screened to eliminate overlapping loci (Table 3). Using 184 isolates obtained between 2007 and 2016, 26 additional loci were obtained by performing two separate multiplex PCR amplifications ( $q 1$ and $q 2$ in Table 1). Together with the reaction mixtures from the previous study, a total of 43 loci were obtained by performing four multiplex PCR amplifications (MLVA43; Table 1).

Comparison of discriminatory power between MLVA and PFGE: The 184 isolates included two O26, two O111, 57 O103, 63 O121, 17 O145, 23 O165, and 20 O91 isolates. MLVA43 and PFGE95 identified 129 and 116 types, respectively, and the D values were 0.991 and 0.971 , respectively (Table 4 ). The discriminatory powers of the two methods were comparable among serogroups O103, O145, O165, and O91. The discriminatory power of MLVA43 for serogroup O121 
Table 1. Primers used in this study

\begin{tabular}{|c|c|c|c|c|c|c|}
\hline $\operatorname{Mix}^{1)}$ & Locus & & Sequence (5'-3') & $\begin{array}{l}\text { Conc. } \\
(\mu \mathrm{M})\end{array}$ & Dye & $\begin{array}{c}\text { Alternative } \\
\text { name }^{2)}\end{array}$ \\
\hline \multirow[t]{21}{*}{$\mathrm{R} 1$} & EH111-8 & $\mathrm{F}$ & CCGGACGAGAGGGAGTAAATGAA & 0.03 & PET & \\
\hline & EH111-8 & $\mathrm{F} 1$ & CCGGGCGAGTAGGAGTAAATGAA & 0.03 & PET & \\
\hline & EH111-8 & $\mathrm{R}$ & CATAAATTATGCTTAATGGAATTAGTAACGCTG & 0.03 & & \\
\hline & EH111-8 & $\mathrm{R} 1$ & CATGAATTATGCTTAATGGAATTAGTCAAGCTG & 0.03 & & \\
\hline & EH157-12 & $\mathrm{F}$ & ACAGTACCCATGCCAGCAA & 0.04 & PET & \\
\hline & EH157-12 & $\mathrm{R}$ & GAAAGCTGGGTGAAAACACCGATGC & 0.04 & & \\
\hline & EHC-1 & $\mathrm{F}$ & GTGCGTAACCTGCTGGCACA & 0.04 & VIC & SVL-3 \\
\hline & EHC-1 & $\mathrm{R}$ & CGCGGCTGCCGGAGTATC & 0.04 & & \\
\hline & EHC-2 & $\mathrm{F}$ & CCAGTTCGGCAGTGAGCTG & 0.04 & VIC & SVL-1 \\
\hline & EHC-2 & $\mathrm{R}$ & ACGCTGGTCCGGGAGATTAT & 0.04 & & \\
\hline & EHC-5 & $\mathrm{F}$ & ATACTACAGACGTCTGCTGATGA & 0.04 & NED & \\
\hline & EHC-5 & $\mathrm{R}$ & CCGCTTTGTTACCGGTCTTTTTC & 0.04 & & \\
\hline & O157-25 & $\mathrm{F}$ & GCCGGAGGAGGGTGATGAGCGGTTATATTTAGTG & 0.04 & PET & \\
\hline & O157-25 & $\mathrm{R}$ & GCGCTGAAAAGACATTCTCTGTTTGGTTTACAC & 0.04 & & \\
\hline & O157-3 & $\mathrm{F}$ & GGCGGTAAGGACAACGGGGTGTTTGAATTG & 0.04 & NED & \\
\hline & O157-3 & $\mathrm{R}$ & GAACAACCTAAAACCCGCCTCGCCATCG & 0.04 & & \\
\hline & O157-34 & $\mathrm{F}$ & TGTTACCAACGCGAAGCTAACAAG & 0.04 & FAM & \\
\hline & O157-34 & $\mathrm{R}$ & AGGCATTAATAGCAGATGTTC & 0.08 & & \\
\hline & O157-9 & $\mathrm{F}$ & GCGCTGGTTTAGCCATCGCCTTCTTCC & 0.04 & VIC & SVL-2 \\
\hline & O157-9 & $\mathrm{R} 1$ & TTCATTAAAATAAAAAATCCCATGGAAAATATTTTTTG & 0.12 & & \\
\hline & O157-9 & $\mathrm{R}$ & GTGTCAGGTGAGCTACAGCCCGCTTACGCTC & 0.08 & & \\
\hline \multirow[t]{16}{*}{$\mathrm{R} 2$} & EH111-11 & $\mathrm{F}$ & GTCAGTAGTTGCGGCTGTAATATTGAAGA & 0.04 & FAM & \\
\hline & EH111-11 & $\mathrm{R}$ & CCTTGTGCATTGAGTTCTGTACATAG & 0.04 & & \\
\hline & EH111-14 & $\mathrm{F}$ & ATGAAATTATCGCAGCATACAATCG & 0.04 & FAM & SVL-10 \\
\hline & EH111-14 & $\mathrm{R}$ & GGGTTTCCATTTTCTTTACCTTCAGG & 0.04 & & \\
\hline & EH26-7 & $\mathrm{F}$ & CССCTATCAAAACTGATACCCGATAAG & 0.08 & PET & \\
\hline & EH26-7 & $\mathrm{R}$ & CGCCGGAAGGCAAAAGATCAT & 0.08 & & \\
\hline & EHC-6 & $\mathrm{F}$ & ATGGAGAACCGTCTGAGTGC & 0.04 & NED & SVL-12 \\
\hline & EHC-6 & $\mathrm{R}$ & TCAGAAATCATCTCCCGGCTCAAC & 0.04 & & \\
\hline & O157-17 & $\mathrm{F}$ & GCAGTTGCTCGGTTTTAACATTGCAGTGATGA & 0.04 & VIC & \\
\hline & O157-17 & $\mathrm{R}$ & AGAAATGGTTTACATGAGTTTGACGATGGCGATC & 0.04 & & \\
\hline & O157-19 & $\mathrm{F}$ & GCAGTGATCATTATTAGCACCGCTTTCTGGATGTTC & 0.04 & NED & \\
\hline & O157-19 & $\mathrm{R}$ & CGGGCAGGGAATAAGGCCACCTGTTAAGC & 0.04 & & \\
\hline & O157-36 & $\mathrm{F}$ & GGCGTCCTTCATCGGCCTGTCCGTTAAAC & 0.04 & NED & \\
\hline & O157-36 & $\mathrm{R}$ & GCCGCTGAAAGCCCACACCATGC & 0.04 & & \\
\hline & O157-37 & $\mathrm{F}$ & AATCAGAGCGGCAGGAAAAAGAAGA & 0.04 & PET & SVL-11 \\
\hline & O157-37 & $\mathrm{R}$ & GGGCTTCTGTCTTTTCAGACCTG & 0.04 & & \\
\hline \multirow[t]{13}{*}{ q1 } & q1701 & $\mathrm{F}$ & TTCATAGTTCAGATCTCAATTTTTCG & 0.04 & FAM & \\
\hline & q1701 & $\mathrm{R}$ & CACGGCTAAACAGTAAACAGTTAATTT & 0.04 & & \\
\hline & q1702 & $\mathrm{F}$ & ACTGCATAGGCACTGATTAACATTC & 0.04 & VIC & \\
\hline & q1702 & $\mathrm{R}$ & GGCAAAAGTGTTTTCTCTTTTTGT & 0.04 & & \\
\hline & q1705 & $\mathrm{F}$ & ATACGCTGAATCGGCTTCC & 0.04 & NED & \\
\hline & q1705 & $\mathrm{R}$ & ACATTGATGTCACGACAGAAGATT & 0.04 & & \\
\hline & q1708 & $\mathrm{F}$ & GCCAGACTATCACTATTGATCTGAAG & 0.04 & FAM & \\
\hline & q1708 & $\mathrm{R}$ & CTAGCATTAACAACAGCACCATCT & 0.04 & & \\
\hline & q1710 & $\mathrm{F}$ & ATTGTCTGCATGTACACCAATTTTT & 0.04 & VIC & \\
\hline & q1710 & $\mathrm{R}$ & ATACGTTGGCTGATAAGACGTTAAA & 0.04 & & \\
\hline & q1712 & $\mathrm{F}$ & ATAGCACATCTGGTAGCTCCTACAC & 0.04 & PET & \\
\hline & q1712 & $\mathrm{R}$ & GAAAGATACCGTTGCAATAATGAAT & 0.04 & & \\
\hline & q1716 & $\mathrm{F}$ & TAGTGCAACTACTTGTGGATAGTGC & 0.04 & VIC & \\
\hline
\end{tabular}




\begin{tabular}{|c|c|c|c|c|c|c|}
\hline $\operatorname{Mix}^{1)}$ & Locus & & Sequence (5'-3') & $\begin{array}{l}\text { Conc. } \\
(\mu \mathrm{M})\end{array}$ & Dye & $\begin{array}{c}\text { Alternative } \\
\text { name }^{2)}\end{array}$ \\
\hline \multirow{40}{*}{$\mathrm{q} 2$} & q1716 & $\mathrm{R}$ & GATCTACCTACCATTGTCATTGGAC & 0.04 & & \\
\hline & q1724 & $\mathrm{F}$ & GTCGTCTGTGGGATGCTCAA & 0.04 & PET & SVL-5 \\
\hline & q1724 & $\mathrm{R}$ & CAGCAATAACAGCAGGACGA & 0.04 & & \\
\hline & $\mathrm{q} 1725$ & $\mathrm{~F}$ & TTCTAAAGGTCTCAATATTGATTTGAATGACG & 0.08 & PET & SVL-6 \\
\hline & q1725 & $\mathrm{R}$ & TGCACCCTCCCATCGTTTCTGTTTCC & 0.08 & & \\
\hline & q1726 & $\mathrm{F}$ & TATCGCATTTTATCGAACATAGTGA & 0.04 & PET & \\
\hline & q1726 & $\mathrm{R}$ & ATCCATGGAGAACACTTTATCTACC & 0.04 & & \\
\hline & $\mathrm{q} 1727$ & $\mathrm{~F}$ & GTAATTGACGACATCGGCTTTAATA & 0.04 & FAM & \\
\hline & q1727 & $\mathrm{R}$ & CTCGCTGGATGATTTTGAATTT & 0.04 & & \\
\hline & q1730 & $\mathrm{F}$ & TTATTTGAACAGAATCTGGAACAGC & 0.04 & NED & \\
\hline & $\mathrm{q} 1730$ & $\mathrm{R}$ & GTAATGCAAAGGACAAAAGAAACAG & 0.04 & & \\
\hline & q1731 & $\mathrm{F}$ & ATTCAGGTAGGTCATGCATAAAAAT & 0.04 & NED & \\
\hline & q1731 & $\mathrm{R}$ & ATGGAGAAAATGTGATGCCTAGAG & 0.04 & & \\
\hline & q1704 & $\mathrm{F}$ & CAGTGTATATCCATCTTCTGGTGAG & 0.08 & FAM & \\
\hline & q1704 & $\mathrm{R}$ & GTATTCAGGTTCGGCTGTCTG & 0.08 & & \\
\hline & q1707 & $\mathrm{F}$ & CGAGCATACTCTGTCTTGTTATGAG & 0.04 & NED & \\
\hline & q1707 & $\mathrm{R}$ & TGAACATACAGGTGGACAACAATAC & 0.04 & & \\
\hline & q1711 & $\mathrm{F}$ & ATATACGGCAGGTGGAGTCATC & 0.04 & FAM & \\
\hline & q1711 & $\mathrm{R}$ & CGGGGACGTGGTTAGTTTTAC & 0.04 & & \\
\hline & q1714 & $\mathrm{F}$ & GTAACAGTGGTAACGAAACGCTCT & 0.04 & VIC & \\
\hline & q1714 & $\mathrm{R}$ & CAGTTTTAACTGCTCATCATTGACA & 0.04 & & \\
\hline & q1715 & $\mathrm{F}$ & TCTATGGTACAGAACCTGTTTTCAC & 0.08 & VIC & \\
\hline & q1715 & $\mathrm{R}$ & GTAAAGCATTGATTTGCCTTCC & 0.08 & & \\
\hline & q1717 & $\mathrm{F}$ & CAACCTTGTTTGTTAAGGTTGAGAT & 0.06 & FAM & \\
\hline & q1717 & $\mathrm{R}$ & GGGATCTCTAATACTGCTGTACGTC & 0.06 & & \\
\hline & q1718 & $\mathrm{F}$ & AGCAAACTGGAACTATCTCAACAAG & 0.04 & VIC & \\
\hline & q1718 & $\mathrm{R}$ & AGTAACAGAGCTCGGTATCTCAATC & 0.04 & & \\
\hline & q1718 & $\mathrm{R} 1$ & CATCTACTCATAGGGGAATTTTGTTC & 0.04 & & \\
\hline & q1720 & $\mathrm{F}$ & GCGAACAGCTGTATCTTAATGAGTT & 0.04 & PET & \\
\hline & q1720 & $\mathrm{R}$ & GAGGATTTAATTTTCCCAACTGTTC & 0.04 & & \\
\hline & q1721 & $\mathrm{F}$ & TTGCAAAGACCATATCAGTGAGTAA & 0.04 & NED & \\
\hline & q1721 & $\mathrm{R}$ & GTTTCGGTAACAGCTTTATGCAG & 0.04 & & \\
\hline & q1722 & $\mathrm{F}$ & ACTATCGTTTTAATAACCGGGATGA & 0.06 & PET & \\
\hline & q1722 & $\mathrm{R}$ & GAAAAAGGCAGTATAAAAACAGCAG & 0.06 & & \\
\hline & q1723 & $\mathrm{F}$ & AAATCGGGCGGGAAGAAG & 0.04 & PET & SVL-23 \\
\hline & q1723 & $\mathrm{R}$ & GGGCGTAAAAAGCAATAAAGG & 0.04 & & \\
\hline & q1728 & $\mathrm{F}$ & ACTTAATCCCTTGAGGAACAGGAGT & 0.04 & NED & \\
\hline & q1728 & $\mathrm{R}$ & GGGAAACTTGACTTCATACTGTGAT & 0.04 & & \\
\hline & q1729 & $\mathrm{F}$ & GTTGTGCTGGGGTAAGAAATGAATA & 0.04 & FAM & \\
\hline & q1729 & $\mathrm{R}$ & GTTCAGTCAGAACGGGACAAGAAT & 0.04 & & \\
\hline
\end{tabular}

\footnotetext{
${ }^{1)}$ : multiplex combination.

${ }^{2)}$ : name in reference 14 .
}

was superior to that of PFGE95.

For further evaluation of the selected loci, the experiment was expanded to a total of 707 isolates. The isolates included 272 O26, 38 O111, 133 O103, $123 \mathrm{O} 121,50 \mathrm{O} 145,31 \mathrm{O} 165$, and $60 \mathrm{O} 91$ isolates. MLVA17 and MLVA43 identified 396 and 468 types, respectively (D values: 0.993 and 0.997 , respectively). The discriminatory powers of MLVA17 and MLVA43 are shown in Table 4, and the D value of each locus is shown in Table 5. The frequency of null alleles at each locus and the allelic ranges are shown in Table 6.
The characteristics of each serogroup or loci were as follows:

O26: The overall discriminatory power of MLVA43 was similar to that of MLVA17. MLVA43 identified more types than did MLVA17. Loci q1704, q1717, q1720, q1722, q1725, EHC-1, EHC-2, EHC-5, EHC6, EH26-7, O157-9, and 0157-37 exhibited moderate to high D values. Loci q1720 and q1722 were highly specific for serogroup $\mathrm{O} 26$ and exhibited low frequency of null alleles.

O111: The overall discriminatory power of MLVA43 
MLVA Scheme for Non-O157 STEC

Table 2. Strains used for sequence screening of VNTR loci

\begin{tabular}{lll}
\hline Escherichia coli serotype, strain & Accession No. & Reference $^{1)}$ \\
\hline O26:H11 str. 11368 & NC_013361.1 & $(19)$ \\
O26:H11 str. FWSEC0001 & CP031922.1 & na \\
O111:H- str. 11128 & NC_013364.1 & $(19)$ \\
O111:H- str. JNE080262 & BLJC01000000 & This study \\
O103:H2 str. 12009 & NC_013353.1 & $(19)$ \\
O103:H2 str. 2015C-3163 & CP027219.1 & $(20)$ \\
O103:H25 str. 2013C-3264 & CP027544.1 & $(20)$ \\
O103:H25 str. NIPH-11060424 & AGSG00000000 & $(21)$ \\
O121:H19 str. FWSEC0006 & CP031910.1 & na \\
O145:H28 str. RM13514 & CP006027.1 & na \\
O165:H25 str. 2013C-4830 & CP027325.1 & $(20)$ \\
O91:H- str. JNE160956 & BLJD01000000 & This study \\
O91:H14 str. 2013C-4404 & CP027376.1 & $(20)$ \\
\hline
\end{tabular}

${ }^{1)}$ : na, not available due to direct submission.

was the same as that of MLVA17. Loci q1702, q1721, q1725, EHC-1, EHC-2, EHC-5, EHC-6, EH111-11, EH111-8, O157-9, and 0157-37 exhibited moderate to high D values. Variations at loci EH111-11, q1702, and q1721 were uniquely observed in serogroup O111. Loci EH111-11 and q1702 were ubiquitously amplified, but locus q1721 exhibited a high frequency of null alleles.

0103: The overall discriminatory power of MLVA43 was similar to that of MLVA17. MLVA43 identified more types than did MLVA17. Loci EHC-2, EH11114, EH111-8, EH26-7, O157-9, O157-19, O157-37, q1701, q1704, q1705, q1716, q1718, q1724, q1726, q1727, q1728, and q1731 exhibited moderate D values; and loci EHC-1, EHC-5, and EHC-6 exhibited high D values. Variations at loci EH111-14, O157-19, q1718, and q1726 were dominantly observed in serogroup O103, though the D values were moderate. Locus q1718 exhibited a very low frequency of null alleles.

0121: The overall discriminatory power of MLVA43 was higher than that of MLVA17. MLVA43 identified more types than did MLVA17. Only three loci, including EHC-1, EHC-2, and EHC-5, exhibited moderate to high D values in MLVA17. In MLVA43, loci q1704, q1705, and q1725 exhibited moderate D values, while locus q1723 exhibited a high D value, which contributed to the high overall discriminatory power of the MLVA43 scheme. Locus q1723 was ubiquitously amplified, but variations at this locus were dominant in serogroup O121.

O145: The overall discriminatory power of MLVA43 was higher than that of MLVA17. MLVA43 identified more types than did MLVA17. Loci EHC-1, EHC-6, O157-9, O157-17, and O157-37 exhibited moderate to high D values in MLVA17. In MLVA43, loci q1704, q1705, q1707, q1708, q1717, and q1727 exhibited moderate D values; while loci q1710 and q1725 exhibited high D values. Loci O157-17, q1707, q1708, and q1710 were specific for serogroup O145 in terms of both variations and frequency of null alleles.

0165: The overall discriminatory power of MLVA43 was similar to that of MLVA17. MLVA43 identified more types than did MLVA17. Loci EHC-5, EHC-6, and 0157-37 exhibited high D values in MLVA17. In
MLVA43, loci q1712 and q1714 exhibited moderate $\mathrm{D}$ values, and loci q1711, q1715, q1716, and q1725 exhibited high D values. Variations at loci q1711, q1714, and q1715 were dominant in serogroup O165. Loci q1711 and q1714 were ubiquitously amplified, and locus q1715 was unique to serogroup O165.

091: The overall discriminatory power of MLVA43 was similar to that of MLVA17. MLVA43 identified more types than did MLVA17. Loci EHC-1, EHC-5, EHC-6, and O157-37 exhibited moderate to high D values in MLVA17. In MLVA43, loci q1704, q1727, and q1730 exhibited moderate D values, and loci q1729 and q1731 exhibited high D values. Variations at loci q1729 and q1730 were observed only in serogroup O91. The frequency of null alleles was low at locus q1729 but high at locus q1730 in serogroup O91.

Ubiquitous or non-functional loci: Loci EHC1, EHC-2, EHC-5, EHC-6, O157-9, O157-37, q1704, q1705, and q1725 exhibited moderate to high D values in multiple serogroups. The D values of loci EH157-12, O157-3, O157-25, and O157-36 were nearly zero. Loci EH157-12 and O157-25 were ubiquitously amplified in most of the serogroups; and the frequency of null alleles at loci O157-3 and O157-36 was almost one in all the serogroups studied.

Analyses of outbreak-related isolates: Isolates related to the two $\mathrm{O} 103$ outbreaks were examined using MLVA43. Outbreaks A and B involved 36 and 18 isolates, respectively. For outbreak A, three MLVA profiles, consisting of 33 isolates, two isolates, and one isolate, were obtained; and they differed from each other at locus O157-9. For outbreak B, two MLVA profiles, one consisting of 17 isolates and the other consisting of one isolate, were obtained; and they differed from each at locus 0157-37.

\section{DISCUSSION}

STEC infection is one of the most serious issues in relation to public health. Molecular subtyping is an essential tool for differentiating isolates and detecting outbreak clusters. MLVA is one of the major tools for subtyping. MLVA is advantageous owing to its rapidity 
Table 3. Characteristics of additional VNTR locus used in this study for non-O157 STEC serogroups

\begin{tabular}{|c|c|c|c|c|c|c|c|c|c|c|}
\hline $\begin{array}{l}\text { Locus } \\
\text { name }\end{array}$ & $\begin{array}{l}\text { Sero- } \\
\text { group }\end{array}$ & Strain ${ }^{1)}$ & $\begin{array}{l}\text { Complete/ } \\
\text { contig No. }\end{array}$ & Start site & Strand & $\begin{array}{l}\text { Predicted } \\
\text { size }\end{array}$ & $\begin{array}{l}\text { Repeat } \\
\text { length }\end{array}$ & Repeat sequence & $\begin{array}{l}\text { Offset } \\
\text { size }\end{array}$ & Putative feature \\
\hline q1701 & $\mathrm{O} 103$ & $2015 \mathrm{C}-3163$ & Complete & 3355162 & $\mathrm{n}$ & 160 & 8 & GCGGGTA & 128 & Intergenic \\
\hline q1702 & $\mathrm{O} 111$ & 11128 & Complete & 2133183 & $\mathrm{n}$ & 260 & 6 & CGCCAG & 248 & MFS transporter $y d h P$ \\
\hline q1704 & O121 & FWSEC0006 & Complete & 2807824 & $\mathrm{c}$ & 500 & 6 & AMAGAW & 440 & Primosomal protein I \\
\hline q1705 & $\mathrm{O} 103$ & $2013 \mathrm{C}-3264$ & Complete & 1312318 & $\mathrm{n}$ & 446 & 12 & WTCTTTATCTGT & 398 & Hypothetical protein \\
\hline q1707 & $\mathrm{O} 145$ & RM13514 & Complete & 1512269 & $\mathrm{n}$ & 342 & 12 & $\begin{array}{l}\text { TCTTTCTTTCAG/ } \\
\text { GATTTCTTTCAG }\end{array}$ & 306 & Hypotheical protein \\
\hline q1708 & $\mathrm{O} 145$ & RM13514 & Complete & 2420529 & $\mathrm{c}$ & 262 & 3 & GCT & 241 & $f l i C$ \\
\hline q1710 & $\mathrm{O} 145$ & RM13514 & Complete & 5470645 & $\mathrm{c}$ & 540 & 24 & $\begin{array}{l}\text { MAACARKYCAWTR } \\
\text { ACARTAYSGWK }\end{array}$ & 348 & Hypotheical protein \\
\hline q1711 & $\mathrm{O} 165$ & $2013 \mathrm{C}-4830$ & Complete & 3882504 & $\mathrm{c}$ & 389 & 7 & GGCTTAT & 361 & Integenic \\
\hline q1712 & O165 & $2013 \mathrm{C}-4830$ & Complete & 1630919 & $\mathrm{c}$ & 490 & 9 & ATTAACGCA & 454 & oxidoreductase \\
\hline q1714 & $\mathrm{O} 165$ & $2013 \mathrm{C}-4830$ & Complete & 3547437 & $\mathrm{n}$ & 328 & 8 & GATGGCGG & 304 & $a d h P$ \\
\hline q1715 & O165 & $2013 \mathrm{C}-4830$ & Complete & 4299938 & $\mathrm{n}$ & 185 & 6 & TCCGGC & 149 & $p d u C$ \\
\hline q1716 & O165 & $2013 \mathrm{C}-4830$ & Complete & 205181 & $\mathrm{c}$ & 154 & 6 & AATGAA & 136 & Intergenic \\
\hline q1717 & $\mathrm{O} 26$ & FWSEC0001 & Complete & 922853 & $\mathrm{c}$ & 166 & 5 & TTTGT & 136 & Hypotheical protein \\
\hline q1718 & O26 & 11368 & Complete & 5583703 & $\mathrm{c}$ & 447 & 8 & TGCGAAGG & 423 & Intergenic \\
\hline q1718 & $\mathrm{O} 103$ & $12009^{2)}$ & Complete & 5290647 & c & 447 & 8 & TGCGAAGG & & \\
\hline q1720 & $\mathrm{O} 26$ & 11368 & Complete & 5509606 & $\mathrm{n}$ & 259 & 8 & RAATATAT & 211 & DNA-binding protein \\
\hline q1721 & O111 & JNE080262 & contig003 & 61797 & $\mathrm{n}$ & 490 & 9 & TGAACCAAC & 454 & Intergenic \\
\hline q1722 & $\mathrm{O} 26$ & 11368 & Complete & 5576065 & $\mathrm{n}$ & 482 & 4 & GCTG & 450 & Intergenic \\
\hline q1723 & O121 & FWSEC0006 & Complete & 4893727 & $\mathrm{c}$ & 418 & 6 & ТСТССС & 364 & Intergenic \\
\hline $\mathrm{q} 1724$ & $\mathrm{O} 103$ & 12009 & Complete & 3051096 & $\mathrm{n}$ & 195 & 6 & GCGCTG & 159 & $h y f F$ \\
\hline q1725 & O103 & 12009 & Complete & 2922510 & $\mathrm{n}$ & 274 & 9 & CAGTTCARC & 220 & $\begin{array}{l}\text { DUF1627 domain- } \\
\text { containing protein }\end{array}$ \\
\hline q1726 & $\mathrm{O} 103$ & $\begin{array}{l}\text { NIPH- } \\
11060424\end{array}$ & contig01055 & 2392 & $\mathrm{c}$ & 348 & 7 & TAAGYAA & 320 & Intergenic \\
\hline q1727 & $\mathrm{O} 103$ & 12009 & Complete & 1020352 & $\mathrm{c}$ & 565 & 39 & $\begin{array}{l}\text { ATMYTGCKGCTGY } \\
\text { GGYGSAACYKGYT } \\
\text { GTTGYGGCTGYTG }\end{array}$ & 292 & $f t s K$ \\
\hline q1728 & $\mathrm{O} 103$ & $\begin{array}{l}\text { NIPH- } \\
11060424\end{array}$ & contig01100 & 82222 & $\mathrm{n}$ & 195 & 13 & TCTCATGGCATGT & 143 & Putative transporter \\
\hline q1729 & O91 & 2013C-4404 & Complete & 17133 & $\mathrm{n}$ & 258 & 6 & GAACAG & 234 & DNA-binding protein \\
\hline q1730 & O91 & JNE160956 & contig024 & 46369 & $\mathrm{c}$ & 352 & 9 & TTGCCCGTA & 298 & No known function \\
\hline q1731 & O91 & 2013C-4404 & Complete & 2851198 & $\mathrm{n}$ & 133 & 8 & ACTACGAA & 117 & Intergenic \\
\hline
\end{tabular}

${ }^{1)}$ : Reference strain for sequence information; start site, strand (normal or complementary), predicted sizes, offset size.

${ }^{2)}$ : The sequence is used for designing R1 primer sequence of q1718.

and high resolution; however, the discriminatory power of MLVA varies among serogroups $(5,14)$. Though the most dominant serogroup in STEC infections is O157, the frequency of detection of non-O157 serogroups have increased in recent years. Among non-O157 serogroups, O26, O111, O103, O121, O45, and $\mathrm{O} 145$ are the most frequently isolated serogroups in the United States. Attempts to expand the target serogroups for MLVA have been reported previously (14).

In Japan, more than 3000 cases of STEC infections are reported annually (2). Serogroups O26, O111, $\mathrm{O} 103, \mathrm{O} 121, \mathrm{O} 145, \mathrm{O} 165$, and $\mathrm{O} 91$ have been regarded important owing to their high frequency of isolation and association with severe cases accompanied by serious conditions, such as HUS. The main aim of this study was to design an MLVA scheme for differentiating serogroups O103, O121, O145, O165, and O91 from the non-O157 STEC isolates obtained from Japan. In our previous study, we designed an MLVA scheme for serogroups O157, O26, and O111 using 17 loci; in the present study, we added 26 new loci to this scheme for identification of the additional serogroups. We used the MLVA43 scheme for evaluating the non-O157 serogroups. The discriminatory power of MLVA43 was 
Table 4. Comparison of discriminatory power of subtyping methods for non-O157 STEC serogroups

\begin{tabular}{|c|c|c|c|c|c|c|}
\hline & Serogroup & $\mathrm{N}$ & Method & \#type & D & $(95 \% \mathrm{CI})$ \\
\hline \multirow[t]{12}{*}{$2007-2016$} & All & 184 & MLVA43 & 129 & 0.991 & $0.987-0.995$ \\
\hline & & & PFGE95 & 116 & 0.971 & $0.956-0.987$ \\
\hline & O103 & 57 & MLVA43 & 39 & 0.976 & $0.956-0.995$ \\
\hline & & & PFGE95 & 46 & 0.992 & $0.987-0.998$ \\
\hline & O121 & 63 & MLVA43 & 37 & 0.954 & $0.926-0.983$ \\
\hline & & & PFGE95 & 19 & 0.769 & $0.674-0.865$ \\
\hline & O145 & 17 & MLVA43 & 11 & 0.882 & $0.750-1.015$ \\
\hline & & & PFGE95 & 12 & 0.956 & 0.913-0.999 \\
\hline & O165 & 23 & MLVA43 & 20 & 0.988 & $0.972-1.004$ \\
\hline & & & PFGE95 & 15 & 0.945 & $0.889-1.000$ \\
\hline & O91 & 20 & MLVA43 & 19 & 0.995 & $0.981-1.008$ \\
\hline & & & PFGE95 & 20 & 1.000 & $\mathrm{na}^{1)}$ \\
\hline \multirow[t]{16}{*}{$2007-2017$} & All & 707 & MLVA17 & 396 & 0.993 & $0.992-0.995$ \\
\hline & & & MLVA43 & 468 & 0.997 & $0.996-0.998$ \\
\hline & $\mathrm{O} 26$ & 272 & MLVA17 & 188 & 0.994 & $0.991-0.996$ \\
\hline & & & MLVA43 & 203 & 0.995 & 0.993-0.997 \\
\hline & O111 & 38 & MLVA17 & 34 & 0.993 & $0.983-1.003$ \\
\hline & & & MLVA43 & 34 & 0.993 & $0.983-1.003$ \\
\hline & O103 & 133 & MLVA17 & 66 & 0.962 & $0.946-0.977$ \\
\hline & & & MLVA43 & 73 & 0.967 & $0.952-0.982$ \\
\hline & O121 & 123 & MLVA17 & 27 & 0.888 & $0.856-0.920$ \\
\hline & & & MLVA43 & 56 & 0.968 & $0.956-0.980$ \\
\hline & O145 & 50 & MLVA17 & 16 & 0.802 & $0.710-0.895$ \\
\hline & & & MLVA43 & 27 & 0.940 & $0.905-0.974$ \\
\hline & O165 & 31 & MLVA17 & 24 & 0.983 & $0.967-0.998$ \\
\hline & & & MLVA43 & 27 & 0.991 & $0.981-1.002$ \\
\hline & O91 & 60 & MLVA17 & 42 & 0.978 & $0.961-0.995$ \\
\hline & & & MLVA43 & 49 & 0.992 & 0.984-0.999 \\
\hline
\end{tabular}

${ }^{1)}$ : not available.

comparable to that of PFGE (for all serogroups except O121), which is the traditional "golden standard" method of molecular subtyping. In serogroup O121, the discriminatory power of MLVA43 was superior to that of PFGE.

As compared to the MLVA17 scheme, MLVA43 scheme identified more profiles in all serogroups except in O111. In serogroups O26 and O111, the MLVA17 scheme could sufficiently differentiate the isolates, as the D values exceeded 0.99 . However, few of the additional loci, including q1725, exhibited high $\mathrm{D}$ values, which can contribute to increasing the discriminatory power of MLVA. Thus, MLVA43 can be used when high discriminatory powers are required for outbreak investigation of serogroups O26 and O111.

In the remaining five serogroups, MLVA43 identified more types than did MLVA17, and raised the D values to $0.94-0.99$. As compared to MLVA 17, MLVA43 dramatically increased the D values for serogroups O121 and O145. Locus q1723 contributed to the increase in the discriminatory power for serogroup O121. This locus corresponds to locus SVL-23 from the previous study, where it was consistently diverse in serogroup O121 (14). Loci O157-17, q1704, q1705, q1707, q1708, q1717, and q1727 were moderately diverse, and locus q1710 was highly diverse in O145. Locus O157-17 was thought to be specific to serogroup O157, but this locus was amplified in almost all O145 isolates. Other loci as well as O157-17 used in this study were likely to have contributed to increasing the discriminatory power for serogroup O145.

Serogroups O165 and O91 were highly differentiated by both MLVA17 and MLVA43. The discriminatory power of MLVA17 was mostly dependent on loci EHC6 and 0157-37. As these loci are known to be located on plasmid(s), MLVA43 should be chosen for routine application $(5,14)$.

Furthermore, among the widely distributed EH11111, EH157-12, EHC-1, O157-34, O157-25, O15719, q1727, q1714, and q1723 loci, loci with moderate or high D values for all tested serogroups, including O157-34 and q1727, may be useful for inter-serogroup differentiation.

The unit length of tandem repeats at loci q1708 and q1722 were 3 and 4, respectively. This may cause problems in inter-laboratory quality assurance owing to the small unit length (18).

The discriminatory powers of few loci, including q1726, were low for intra- and inter-serogroups. Although loci with moderate and high D values for 
Table 5. D values of VNTR loci for non-O157 STEC serogroups

\begin{tabular}{|c|c|c|c|c|c|c|c|c|}
\hline Locus & All & $\mathrm{O} 26$ & O111 & O103 & O121 & $\mathrm{O} 145$ & O165 & O91 \\
\hline EHC-1 & 0.885 & 0.805 & 0.912 & 0.790 & 0.760 & 0.544 & 0.000 & 0.903 \\
\hline EHC-2 & 0.898 & 0.918 & 0.853 & 0.298 & 0.584 & 0.079 & 0.000 & 0.000 \\
\hline EHC-5 & 0.486 & 0.599 & 0.292 & 0.606 & 0.183 & 0.040 & 0.738 & 0.333 \\
\hline EHC-6 & 0.605 & 0.411 & 0.198 & 0.618 & 0.095 & 0.224 & 0.912 & 0.739 \\
\hline EH26-7 & 0.562 & 0.681 & 0.000 & 0.127 & 0.000 & 0.000 & 0.000 & 0.033 \\
\hline EH111-8 & 0.285 & 0.000 & 0.337 & 0.180 & 0.016 & 0.040 & 0.000 & 0.000 \\
\hline EH111-11 & 0.101 & 0.000 & 0.511 & 0.000 & 0.000 & 0.000 & 0.000 & 0.000 \\
\hline EH111-14 & 0.478 & 0.007 & 0.053 & 0.190 & 0.000 & 0.040 & 0.000 & 0.066 \\
\hline EH157-12 & 0.006 & 0.000 & 0.000 & 0.000 & 0.000 & 0.040 & 0.065 & 0.000 \\
\hline O157-3 & 0.000 & 0.000 & 0.000 & 0.000 & 0.000 & 0.000 & 0.000 & 0.000 \\
\hline O157-9 & 0.690 & 0.769 & 0.882 & 0.277 & 0.000 & 0.389 & 0.000 & 0.000 \\
\hline O157-17 & 0.125 & 0.000 & 0.000 & 0.000 & 0.000 & 0.259 & 0.000 & 0.000 \\
\hline O157-19 & 0.039 & 0.000 & 0.000 & 0.153 & 0.000 & 0.040 & 0.000 & 0.000 \\
\hline O157-25 & 0.000 & 0.000 & 0.000 & 0.000 & 0.000 & 0.000 & 0.000 & 0.066 \\
\hline O157-34 & 0.481 & 0.000 & 0.000 & 0.127 & 0.000 & 0.040 & 0.000 & 0.000 \\
\hline O157-36 & 0.000 & 0.000 & 0.000 & 0.000 & 0.000 & 0.000 & 0.000 & 0.000 \\
\hline O157-37 & 0.412 & 0.312 & 0.910 & 0.325 & 0.080 & 0.154 & 0.531 & 0.550 \\
\hline q1701 & 0.100 & 0.000 & 0.000 & 0.413 & 0.000 & 0.040 & 0.000 & 0.000 \\
\hline q1702 & 0.047 & 0.029 & 0.336 & 0.087 & 0.000 & 0.000 & 0.000 & 0.000 \\
\hline q1704 & 0.672 & 0.420 & 0.104 & 0.233 & 0.364 & 0.150 & 0.065 & 0.381 \\
\hline q1705 & 0.636 & 0.118 & 0.053 & 0.423 & 0.222 & 0.190 & 0.065 & 0.000 \\
\hline q1707 & 0.122 & 0.000 & 0.000 & 0.000 & 0.000 & 0.187 & 0.000 & 0.000 \\
\hline q1708 & 0.125 & 0.000 & 0.000 & 0.000 & 0.000 & 0.224 & 0.000 & 0.000 \\
\hline q1710 & 0.127 & 0.000 & 0.000 & 0.000 & 0.000 & 0.633 & 0.000 & 0.000 \\
\hline q1711 & 0.085 & 0.000 & 0.000 & 0.000 & 0.000 & 0.000 & 0.512 & 0.000 \\
\hline q1712 & 0.538 & 0.000 & 0.000 & 0.140 & 0.000 & 0.078 & 0.469 & 0.033 \\
\hline q1714 & 0.098 & 0.036 & 0.000 & 0.000 & 0.016 & 0.000 & 0.443 & 0.000 \\
\hline q1715 & 0.359 & 0.000 & 0.000 & 0.000 & 0.000 & 0.040 & 0.647 & 0.000 \\
\hline q1716 & 0.413 & 0.007 & 0.000 & 0.178 & 0.016 & 0.040 & 0.787 & 0.000 \\
\hline q1717 & 0.096 & 0.194 & 0.000 & 0.000 & 0.000 & 0.246 & 0.000 & 0.000 \\
\hline q1718 & 0.518 & 0.106 & 0.000 & 0.193 & 0.000 & 0.040 & 0.000 & 0.000 \\
\hline q1720 & 0.587 & 0.684 & 0.000 & 0.130 & 0.000 & 0.000 & 0.065 & 0.000 \\
\hline q1721 & 0.020 & 0.000 & 0.329 & 0.000 & 0.000 & 0.000 & 0.000 & 0.000 \\
\hline q1722 & 0.488 & 0.755 & 0.053 & 0.000 & 0.000 & 0.000 & 0.000 & 0.000 \\
\hline q1723 & 0.281 & 0.007 & 0.000 & 0.000 & 0.858 & 0.000 & 0.000 & 0.033 \\
\hline q1724 & 0.305 & 0.007 & 0.053 & 0.377 & 0.000 & 0.079 & 0.000 & 0.000 \\
\hline q1725 & 0.755 & 0.656 & 0.717 & 0.059 & 0.212 & 0.766 & 0.763 & 0.033 \\
\hline q1726 & 0.036 & 0.000 & 0.000 & 0.158 & 0.000 & 0.079 & 0.000 & 0.000 \\
\hline q1727 & 0.563 & 0.007 & 0.000 & 0.167 & 0.079 & 0.154 & 0.000 & 0.239 \\
\hline q1728 & 0.513 & 0.000 & 0.000 & 0.283 & 0.000 & 0.079 & 0.000 & 0.033 \\
\hline q1729 & 0.103 & 0.000 & 0.000 & 0.000 & 0.000 & 0.040 & 0.000 & 0.666 \\
\hline q1730 & 0.025 & 0.000 & 0.000 & 0.000 & 0.000 & 0.000 & 0.000 & 0.271 \\
\hline q1731 & 0.486 & 0.029 & 0.000 & 0.320 & 0.000 & 0.078 & 0.000 & 0.505 \\
\hline
\end{tabular}


MLVA Scheme for Non-O157 STEC

Table 6. Frequency of null allele (\%) and range of alleles

\begin{tabular}{|c|c|c|c|c|c|c|c|c|c|}
\hline & All & $\mathrm{O} 26$ & O111 & O103 & O121 & $\mathrm{O} 145$ & O165 & O91 & Allelic range \\
\hline EHC-1 & 0 & 0 & 0 & 1 & 0 & 2 & 0 & 0 & $3-28$ \\
\hline EHC-2 & 11 & 0 & 3 & 0 & 0 & 96 & 100 & 0 & $3-35$ \\
\hline EHC-5 & 71 & 61 & 84 & 62 & 90 & 98 & 42 & 82 & $2-25$ \\
\hline EHC-6 & 59 & 76 & 8 & 29 & 95 & 88 & 0 & 17 & $3-33$ \\
\hline ЕH26-7 & 63 & 6 & 100 & 93 & 100 & 100 & 100 & 98 & $1-6$ \\
\hline EH111-8 & 9 & 0 & 0 & 1 & 1 & 0 & 0 & 100 & $1-13$ \\
\hline EH111-11 & 0 & 0 & 0 & 0 & 0 & 0 & 0 & 0 & $2-4$ \\
\hline EH111-14 & 39 & 0 & 0 & 11 & 100 & 98 & 100 & 97 & $1-2$ \\
\hline EH157-12 & 0 & 0 & 0 & 0 & 0 & 0 & 3 & 0 & $1-2$ \\
\hline O157-3 & 100 & 100 & 100 & 100 & 100 & 100 & 100 & 100 & $\mathrm{na}^{1)}$ \\
\hline O157-9 & 52 & 0 & 0 & 85 & 100 & 78 & 100 & 100 & $5-21$ \\
\hline O157-17 & 93 & 100 & 100 & 100 & 100 & 6 & 100 & 100 & $3-5$ \\
\hline O157-19 & 0 & 0 & 0 & 0 & 0 & 0 & 0 & 0 & $0-7$ \\
\hline O157-25 & 0 & 0 & 0 & 0 & 0 & 0 & 0 & 0 & 2 \\
\hline O157-34 & 0 & 0 & 0 & 0 & 0 & 2 & 0 & 0 & $1-3$ \\
\hline O157-36 & 100 & 100 & 100 & 100 & 100 & 100 & 100 & 100 & $\mathrm{na}^{1)}$ \\
\hline O157-37 & 76 & 83 & 13 & 82 & 96 & 92 & 68 & 23 & $0-18$ \\
\hline q1701 & 95 & 100 & 100 & 73 & 100 & 98 & 100 & 100 & $3-7$ \\
\hline q1702 & 0 & 0 & 0 & 0 & 0 & 0 & 0 & 0 & $2-9$ \\
\hline q1704 & 45 & 16 & 95 & 87 & 2 & 92 & 97 & 75 & $1-12$ \\
\hline q1705 & 47 & 94 & 0 & 2 & 9 & 4 & 0 & 100 & $2-5$ \\
\hline q1707 & 93 & 100 & 100 & 100 & 100 & 8 & 100 & 100 & $3-4$ \\
\hline q1708 & 93 & 100 & 100 & 100 & 100 & 6 & 100 & 100 & $6-8$ \\
\hline q1710 & 93 & 100 & 100 & 100 & 100 & 6 & 100 & 100 & $3-11$ \\
\hline q1711 & 0 & 0 & 0 & 0 & 0 & 0 & 0 & 0 & $2-5$ \\
\hline q1712 & 52 & 100 & 100 & 8 & 0 & 96 & 0 & 2 & $2-5$ \\
\hline q1714 & 0 & 1 & 0 & 0 & 1 & 0 & 0 & 0 & $2-4$ \\
\hline q1715 & 78 & 100 & 100 & 100 & 0 & 98 & 0 & 100 & $2-7$ \\
\hline q1716 & 23 & 0 & 100 & 10 & 1 & 98 & 0 & 100 & $2-8$ \\
\hline q1717 & 95 & 90 & 100 & 100 & 100 & 86 & 100 & 100 & $3-8$ \\
\hline q1718 & 45 & 1 & 100 & 9 & 100 & 98 & 100 & 100 & $2-5$ \\
\hline q1720 & 60 & 0 & 100 & 93 & 100 & 100 & 97 & 100 & $1-12$ \\
\hline q1721 & 99 & 100 & 82 & 100 & 100 & 100 & 100 & 100 & $3-5$ \\
\hline q1722 & 69 & 21 & 97 & 100 & 100 & 100 & 100 & 100 & $2-24$ \\
\hline q1723 & 0 & 0 & 0 & 0 & 0 & 0 & 0 & 0 & $3-15$ \\
\hline q1724 & 2 & 0 & 0 & 8 & 0 & 2 & 0 & 0 & $2-7$ \\
\hline q1725 & 37 & 15 & 50 & 97 & 1 & 30 & 0 & 98 & $2-12$ \\
\hline q1726 & 98 & 100 & 100 & 92 & 100 & 96 & 100 & 100 & $5-16$ \\
\hline q1727 & 0 & 0 & 0 & 0 & 0 & 0 & 0 & 0 & $1-9$ \\
\hline q1728 & 54 & 100 & 100 & 8 & 0 & 2 & 0 & 98 & $1-4$ \\
\hline q1729 & 95 & 100 & 100 & 100 & 100 & 98 & 100 & 38 & $2-4$ \\
\hline q1730 & 99 & 100 & 100 & 100 & 100 & 100 & 100 & 85 & $3-8$ \\
\hline q1731 & 29 & 1 & 0 & 19 & 100 & 4 & 100 & 35 & $1-11$ \\
\hline
\end{tabular}

${ }^{1)}$ : not available. 
each serogroup can be selected for analizing specific serogroups at a low cost, other options need to be considered. For example, q1726 was designed using the sequence of strain NIPH-11060424, which originated in Norway with serotype $\mathrm{O} 103: \mathrm{H} 25$. H25 is a minor $\mathrm{H}$-type of $\mathrm{O} 103$ in Japan, while $\mathrm{H} 2$ is the dominant H-type. It is plausible that q1726 could exhibit high discriminatory power in settings where O103:H25 is more abundant. Thus, loci with low discriminatory powers should be evaluated in different settings to determine their significance.

The MLVA scheme developed in this study allowed discrimination among serogroups O103, O121, O145, O165, and O91 as well as $\mathrm{O} 26$ and O111. Very few single-locus variations were detected in two sets of outbreak-related isolates. Furthermore, the number of isolates tested in this study might be less for a comprehensive evaluation; therefore, in future, this scheme should be tested using diverse isolates. Applicability of this scheme in outbreak investigation can also be evaluated using a large panel of isolates. Nevertheless, the scheme would be useful for routine molecular subtyping of STEC. MLVA is rapid and cost-efficient for routine testing (6). The serogroups O157, O26, O111, O103, O121, O145, O165, and O91 cover approximately $90 \%$ of the STEC isolates in Japan. MLVA17 and MLVA43 schemes are easy to perform and involve only two and four multiplex PCR amplifications, respectively.

Acknowledgments We thank Yukinari Saitoh, Ayumi Takemoto, Yukie Nakajima for technical assistance. We thank the staff of municipal and prefectural public health institutes for providing the isolates for MLVA and PFGE. We thank Sumie Kobori, Koji Kikuchi, Kentaro Tomari, Hiroyuki Ueno of Saitama City Institute of Health Science and Research, Japan, and Hideyuki Kariya of Okayama Prefectural Institute for Environmental Science and Public Health, Okayama, Japan, for providing the isolates for WGS. This work was partly supported by grants-inaid from the Ministry of Health, Labour, and Welfare of Japan (H30-Shinkogyosei-Ippan-001 and H29-Shokuhin-Ippan-001).

Conflict of interest None to declare.

\section{REFERENCES}

1. Tarr PI, Gordon CA, Chandler WL. Shiga-toxin-producing Escherichia coli and haemolytic uraemic syndrome. Lancet. 2005;365:1073-86.

2. National Institute of Infectious Diseases and Tuberculosis and Infectious Diseases Control Division, Ministry of Health, Labour and Welfare of Japan. Enterohemorrhagic Escherichia coli (EHEC) infection, as of March 2019, Japan. Infect Agents Surveillance Rep. 2019;40:71-2.

3. Karmali MA, Gannon V, Sargeant JM. Verocytotoxin-producing Escherichia coli (VTEC). Vet Microbiol. 2010;140:360-70.

4. Swaminathan B, Barrett TJ, Hunter SB, et al. PulseNet: the molecular subtyping network for foodborne bacterial disease surveillance, United States. Emerg Infect Dis. 2001;7:382-89.

5. Izumiya H, Pei Y, Terajima J, et al. New system for multilocus variable-number tandem-repeat analysis of the enterohemorrhagic Escherichia coli strains belonging to three major serogroups: O157, O26, and O111. Microbiol Immunol. 2010;54:569-77.

6. Lee $\mathrm{K}$, Izumiya $\mathrm{H}$, Iyoda $\mathrm{S}$, et al. Effective surveillance using multilocus variable-number tandem-repeat analysis and wholegenome sequencing for enterohemorrhagic Escherichia coli O157. Appl Environ Microbiol. 2019;85:e00728-19.

7. van Belkum A. Tracing isolates of bacterial species by multilocus variable number of tandem repeat analysis (MLVA). FEMS Immunol Med Microbiol. 2007;49:22-27.

8. Hyytiä-Trees E, Smole SC, Fields PA, et al. Second generation subtyping: a proposed PulseNet protocol for multiple-locus variable-number tandem repeat analysis of Shiga toxin-producing Escherichia coli O157 (STEC O157). Foodborne Pathog Dis. 2006;3:118-31.

9. Lindstedt B-A, Vardund T, Aas L, et al. Multiple-locus variablenumber tandem-repeats analysis of Salmonella enterica subsp. enterica serovar Typhimurium using PCR multiplexing and multicolor capillary electrophoresis. J Microbiol Methods. 2004;59:163-72.

10. Larsson JT, Torpdahl M, Petersen RF, et al. Development of a new nomenclature for Salmonella Typhimurium multilocus variable number of tandem repeats analysis (MLVA). Euro Surveill. 2009;14:19174.

11. Hopkins KL, Peters TM, Pinna E de, et al. Standardisation of multilocus variable-number tandem-repeat analysis (MLVA) for subtyping of Salmonella enterica serovar Enteritidis. Euro Surveill. 2011;16:19942.

12. Liang S-Y, Watanabe H, Terajima J, et al. Multilocus variablenumber tandem-repeat analysis for molecular typing of Shigella sonnei. J Clin Microbiol. 2007:45:3574-80.

13. Danin-Poleg Y, Cohen LA, Gancz H, et al. Vibrio cholerae strain typing and phylogeny study based on simple sequence repeats. J Clin Microbiol. 2007:45:736-46.

14. Timmons C, Trees E, Ribot EM, et al. Multiple-locus variablenumber tandem repeat analysis for strain discrimination of non-O157 Shiga toxin-producing Escherichia coli. J Microbiol Methods. 2016;125:70-80.

15. Ribot EM, Fair MA, Gautom R, et al. Standardization of pulsed-field gel electrophoresis protocols for the subtyping of Escherichia coli O157:H7, Salmonella, and Shigella for PulseNet. Foodborne Pathog Dis. 2006;3:59-67.

16. Grundmann H, Hori S, Tanner G. Determining confidence intervals when measuring genetic diversity and the discriminatory abilities of typing methods for microorganisms. J Clin Microbiol. 2001;39:4190-2.

17. Bankevich A, Nurk S, Antipov D, et al. SPAdes: a new genome assembly algorithm and its applications to single-cell sequencing. J Comput Biol. 2012;19:455-77.

18. Nadon CA, Trees E, Ng LK, et al. Development and application of MLVA methods as a tool for inter-laboratory surveillance. Eurosurveillance. 2013;18:20565.

19. Ogura Y, Ooka T, Iguchi A, et al. Comparative genomics reveal the mechanism of the parallel evolution of O157 and non-O157 enterohemorrhagic Escherichia coli. Proc Natl Acad Sci USA. 2009; 106:17939-44

20. Patel PN, Lindsey RL, Garcia-Toledo L, et al. High-quality whole-genome sequences for 77 Shiga toxin-producing Escherichia coli strains generated with PacBio sequencing. Genome Announc. 2018;6:e00391-18.

21. L'Abée-Lund TM, Jørgensen HJ, O'Sullivan K, et al. The highly virulent 2006 Norwegian EHEC O103:H25 outbreak strain is related to the 2011 German O104:H4 outbreak strain. PLoS ONE. 2012;7:e31413 